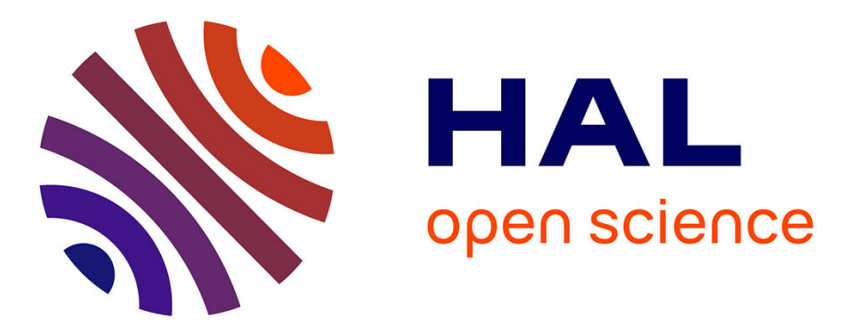

\title{
Suppression of thermal conductivity by rattling modes in thermoelectric sodium cobaltate
}

\author{
D. J. Voneshen, K. Refson, E. Borissenko, M. Krisch, A. Bosak, A. Piovano, \\ E. Cemal, M. Enderle, M. Gutmann, M. Hoesch, et al.
}

\section{- To cite this version:}

D. J. Voneshen, K. Refson, E. Borissenko, M. Krisch, A. Bosak, et al.. Suppression of thermal conductivity by rattling modes in thermoelectric sodium cobaltate. Nature Materials, 2013, 12 (11), pp.1028 - 1032. 10.1038/nmat3739 . cea-01411905

\section{HAL Id: cea-01411905 https://hal-cea.archives-ouvertes.fr/cea-01411905}

Submitted on 7 Dec 2016

HAL is a multi-disciplinary open access archive for the deposit and dissemination of scientific research documents, whether they are published or not. The documents may come from teaching and research institutions in France or abroad, or from public or private research centers.
L'archive ouverte pluridisciplinaire HAL, est destinée au dépôt et à la diffusion de documents scientifiques de niveau recherche, publiés ou non, émanant des établissements d'enseignement et de recherche français ou étrangers, des laboratoires publics ou privés. 


\title{
Suppression of thermal conductivity by rattling modes in thermoelectric sodium cobaltate
}

\author{
D. J. Voneshen ${ }^{1}$, K. Refson ${ }^{2}$, E. Borissenko ${ }^{3}$, M. Krisch ${ }^{3}$, A. Bosak ${ }^{3}$, A. Piovano ${ }^{4}$, E. Cemal ${ }^{1,4}$, \\ M. Enderle ${ }^{4}$, M. J. Gutmann ${ }^{5}$, M. Hoesch ${ }^{6}$, M. Roger ${ }^{7}$, L. Gannon ${ }^{8}$, A. T. Boothroyd ${ }^{8}$, S. Uthayakumar ${ }^{1}$, \\ D. G. Porter ${ }^{1}$ and J. P. Goff ${ }^{1 \star}$
}

\begin{abstract}
The need for both high electrical conductivity and low thermal conductivity creates a design conflict for thermoelectric systems, leading to the consideration of materials with complicated crystal structures'. Rattling of ions in cages results in low thermal conductivity ${ }^{2-5}$, but understanding the mechanism through studies of the phonon dispersion using momentumresolved spectroscopy is made difficult by the complexity of the unit cells ${ }^{6}$. We have performed inelastic X-ray and neutron scattering experiments that are in remarkable agreement with our first-principles density-functional calculations of the phonon dispersion for thermoelectric $\mathrm{Na}_{0.8} \mathrm{CoO}_{2}$, which has a large-period superstructure ${ }^{7}$. We have directly observed an Einstein-like rattling mode at low energy, involving large anharmonic displacements of the sodium ions inside multi-vacancy clusters. These rattling modes suppress the thermal conductivity by a factor of six compared with vacancy-free $\mathrm{NaCoO}_{2}$. Our results will guide the design of the next generation of materials for applications in solid-state refrigerators and power recovery.

New thermoelectric materials often have large unit cells and, therefore, calculations of the thermal conductivity, $\kappa$, using acoustic phonon branches within the usual Debye framework are inadequate. Instead, it is necessary to take account of the multitude of optical modes and, therefore, detailed knowledge of the phonon dispersion is required. The ability to measure phonon dispersions using inelastic X-ray scattering (IXS) and inelastic neutron scattering (INS) provides unprecedented opportunities to understand thermal transport in these systems. These experiments on samples with a complicated unit cell require a model calculation to assign spectral features. For complex unit cells ab initio calculations are also demanding, and still require experimental validation ${ }^{6}$.
\end{abstract}

The efficiency of operation of a thermoelectric material is usually measured by its figure-of-merit, $Z T=T S^{2} \sigma / \kappa$, a dimensionless number proportional to the square of the Seebeck coefficient, $S$, and the ratio $(\sigma / \kappa)$ of the electrical to thermal conductivities. The phonon-glass electron-crystal model ${ }^{2}$ has been proposed, where cages form regular periodic crystal lattices in which electrons or holes can move freely, but the rattling of loosely bound atoms in the cage is believed to disrupt the vibrational modes of the cage so that heat conduction is comparable to glasses ${ }^{3-5}$. However, other theoretical studies have called into question the validity of the phonon-glass electron-crystal and rattler concepts ${ }^{8,9}$ and, moreover, INS measurements demonstrate that phonon excitations are sharp for these materials and are certainly not glass like ${ }^{10,11}$. The presence of a low-energy rattling mode can lead to an avoided crossing with acoustic phonons. Although such an avoided crossing is suggestive of the presence of a rattler, it is not proof, as is confirmed by the fact that it is observed on some occasions, but not others ${ }^{11}$.

Particularly good thermoelectric properties have been observed for sodium cobaltate ${ }^{12-15}$. Theoretical studies have considered the electronic contributions to $Z T$ using density functional theory (DFT) and Boltzmann transport theory ${ }^{16}$, explaining the suppression of $S$ in large magnetic fields ${ }^{13}$. The phonon dispersion for stoichiometric $\mathrm{NaCoO}_{2}$ has been calculated using DFT (refs 17,18) and the results are broadly consistent with Raman ${ }^{19-21}$, infrared $^{22}$ and IXS (ref. 23) experiments. However, thermoelectric $\mathrm{Na}_{x} \mathrm{CoO}_{2}$ contains multi-vacancy clusters that order to form longranged superstructures ${ }^{7}$ (Fig. 1a) and those calculations omit the low-energy rattling modes caused by the multi-vacancy clusters. Here we have included the multi-vacancy clusters in the calculation of the phonon dispersion and compared it with measurements of momentum-resolved IXS and INS spectra from single-crystal $\mathrm{Na}_{0.8} \mathrm{CoO}_{2}$ with a well-defined superstructure. We are able to unambiguously identify an Einstein-like rattler mode at low energy and, as a result, to quantitatively account for the suppression of $\kappa$ for this class of materials.

IXS measurements were performed on a single crystal of $\mathrm{Na}_{0.8} \mathrm{CoO}_{2}$ of dimension a few hundred micrometres using the ID28 spectrometer at the European Synchrotron Radiation Facility. The sample was cooled to a temperature $T=200 \mathrm{~K}$, where it was found to be predominantly in the square lattice tri-vacancy phase ${ }^{7}$. We also performed measurements at elevated temperature, at $T=320 \mathrm{~K}$ in the disordered stripe phase ${ }^{24}$, and at $T=400 \mathrm{~K}$ in the disordered phase. High-resolution INS measurements were performed on a larger crystal using the triple-axis spectrometer IN8 at the Institut Laue-Langevin, and phonon lifetimes were determined as a function of temperature in the square tri-vacancy phase.

Figure 1 shows the X-ray intensity as a function of energy transfer with a fixed wavevector transfer, $\mathbf{Q}$, at $T=200 \mathrm{~K}$. The solid lines show the results of first-principles lattice dynamics calculations using the CASTEP $\operatorname{code}^{25}$, for the large-period square

\footnotetext{
${ }^{1}$ Department of Physics, Royal Holloway, University of London, Egham TW20 OEX, UK, ${ }^{2}$ Science and Technology Facilities Council, Rutherford Appleton Laboratory, Harwell Science and Innovation Campus, Didcot OX11 0QX, UK, ${ }^{3}$ European Synchrotron Radiation Facility, 6 Rue Jules Horowitz, BP 220, 38043 Grenoble, Cedex 9, France, ${ }^{4}$ Institut Laue-Langevin, 156X, 38042 Grenoble Cedex, France, ${ }^{5}$ ISIS Facility, Rutherford Appleton Laboratory, Chilton, Didcot OX11 OQX, UK, ${ }^{6}$ Diamond Light Source, Harwell Science and Innovation Campus, Didcot OX11 ODE, UK, ${ }^{7}$ Service de Physique de I'Etat Condensé, (CNRS/MIPPU/URA 2464), DSM/DRECAM/SPEC, CEA Saclay, P.C. 135, F-91191 Gif Sur Yvette, France, ${ }^{8}$ Department of Physics, Oxford University, Oxford OX1 3PU, UK. *e-mail: Jon.Goff@rhul.ac.uk
} 


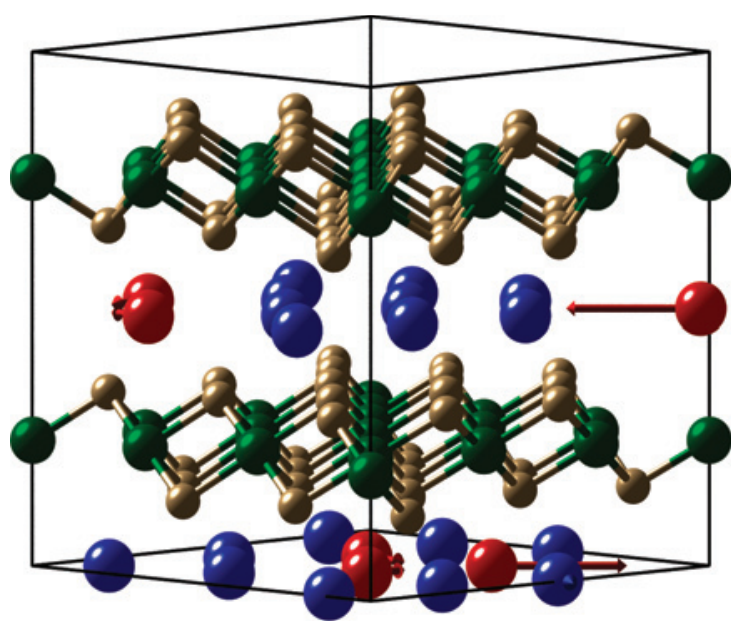

b

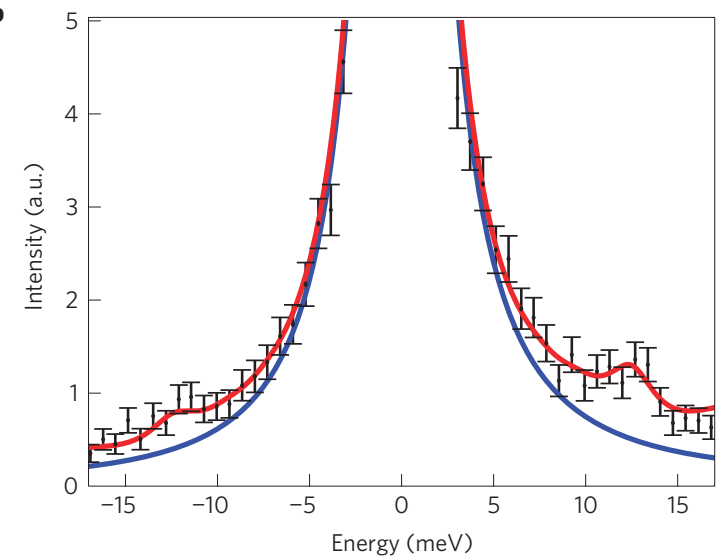

c

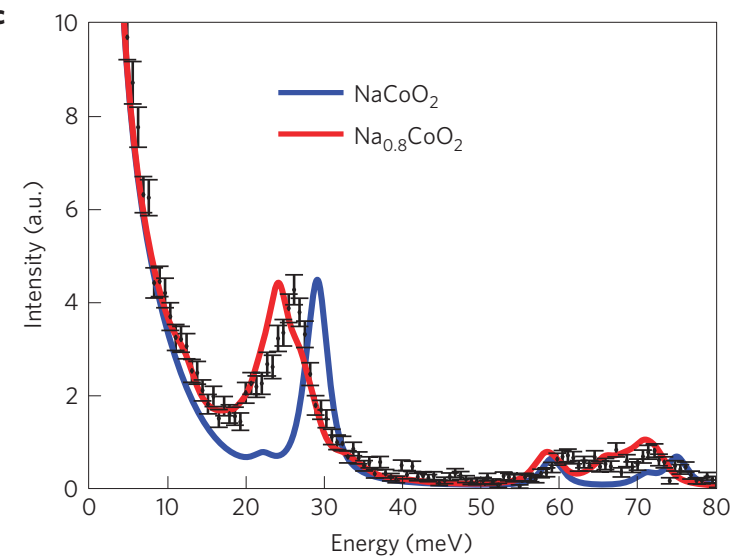

Figure 1 | Einstein-like rattling modes in sodium cobaltate. a, The supercell of $\mathrm{Na}_{0.80} \mathrm{CoO}_{2}$ showing the rattling mode at $E \sim 13$ meV comprising mainly displacements of the (red) $\mathrm{Na} 2 b$ ions inside tri-vacancy clusters. $\mathrm{Na} 2 d$ ions are blue, $\mathrm{Co}$ is green and $\mathrm{O}$ is gold. The length of the arrow indicates the relative amplitude for each ion, not an absolute displacement. $\mathbf{b}$, IXS at $\mathbf{Q}=(1.25,1.25,1)$ measured from $\mathrm{Na}_{0.80} \mathrm{CoO}_{2}$ at $T=200 \mathrm{~K}$, focusing on the rattling mode in $\mathbf{a}$. The red line shows the CASTEP calculation for the $\mathrm{Na}_{0.80} \mathrm{CoO}_{2}$ superstructure comprising a square array of tri-vacancy clusters, and the blue line shows the corresponding calculation for $\mathrm{NaCoO}_{2}$. c, IXS at $\mathbf{Q}=(1.17,0,0)$ from $\mathrm{Na}_{0.80} \mathrm{CoO}_{2}$ at $T=200 \mathrm{~K}$ showing typical data for the full spectrum. Error bars are determined by Poisson statistics.

superstructure determined by X-ray diffraction and for a lattice of $\mathrm{NaCoO}_{2}$ with no vacancies. The latter calculation used the primitive cell of space group $\mathrm{P}_{3} / \mathrm{mmc}$ with $\mathrm{CoO}_{2}$ layers of edge-sharing $\mathrm{CoO}_{6}$ octahedra and two Wyckoff sites, $2 b$ and $2 d$, available for

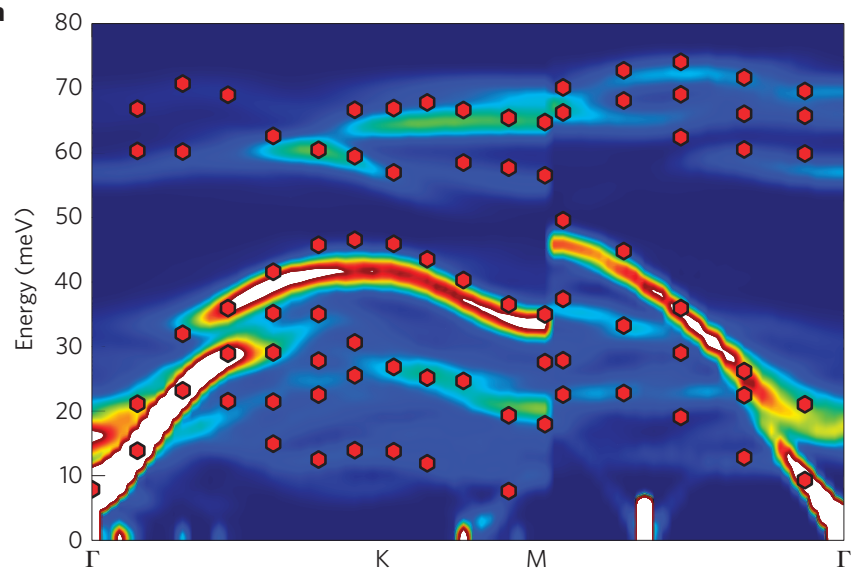

b

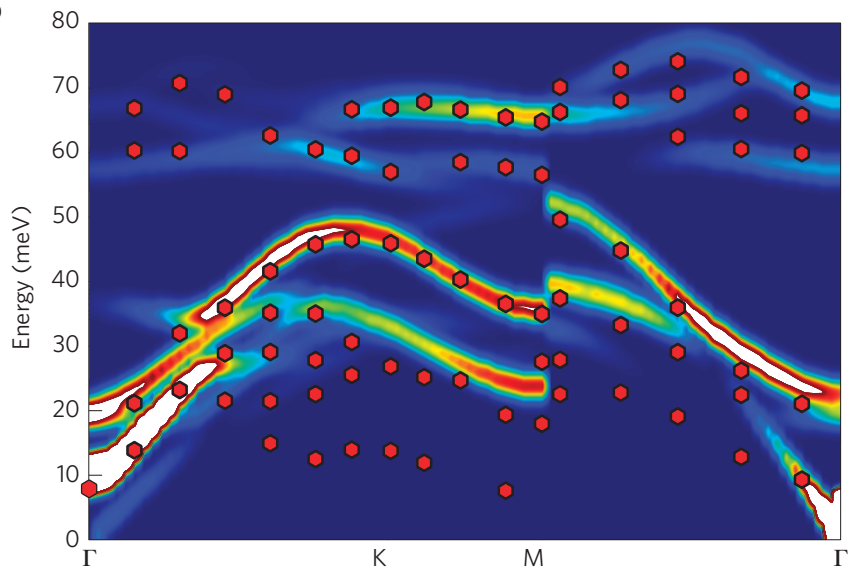

Figure $\mathbf{2}$ | Phonon dispersion of sodium cobaltate. $\mathbf{a}, \mathbf{b}$, CASTEP

calculations for the $\mathrm{Na}_{0.8} \mathrm{CoO}_{2}$ superstructure comprising a square array of tri-vacancy clusters (a) and for $\mathrm{NaCoO}_{2}$ (b), shown as a colour contour diagram on a hot scale (blue, low intensity; white, high intensity). The experimental data obtained for $\mathrm{Na}_{0.8} \mathrm{CoO}_{2}$ at $T=200 \mathrm{~K}$ are shown as red hexagons. The superstructure is essential to capture the scattering at lower energies below $E \sim 20 \mathrm{meV}$.

$\mathrm{Na}$, and we find consistency with previous calculations for this structure $^{17,18}$. It is clear that the calculated spectrum of the square phase differs markedly from that of the stoichiometric compound at energies below $E \sim 20 \mathrm{meV}$; in particular there is an additional narrow mode at $E \sim 13 \mathrm{meV}$ in the superstructure that is completely absent in stoichiometric $\mathrm{NaCoO}_{2}$.

Scans of variable energy transfer were performed at a number of settings of $\mathbf{Q}$ along the high-symmetry directions $\Gamma \rightarrow \mathrm{K} \rightarrow \mathrm{M}$ from (111) and for $\Gamma \rightarrow M$ from (100), and the fitted peak positions are shown in Fig. 2 as red hexagons. The calculated scattering intensity is shown as colour contour diagrams for the square superstructure and the ideal compound $\mathrm{NaCoO}_{2}$. The agreement between the $a b$ initio calculations for the square superstructure and the experimental data is remarkable. The mode at $E \sim 13 \mathrm{meV}$ is captured by the model, and a typical scan is shown in Fig. 1b. In contrast, the ideal vacancy-free compound has no modes below $E \sim 20 \mathrm{meV}$ away from the zone centre.

It is possible to trace the mode at $E \sim 13 \mathrm{meV}$ back to the zone centre, and the relative amplitudes of the ionic displacements are shown in Fig. 1a. The blue spheres in Fig. 1a are the $\mathrm{Na} 2 d$ sites, and these are fully occupied in the stoichiometric compound. The red spheres are on the inequivalent $\mathrm{Na} 2 b$ sites and, because they are associated with clusters of three vacancies, they are expected to be more loosely bound. The atomic displacement pattern is 
characterized by a predominant in-plane amplitude of vibration of the $\mathrm{Na} 2 b$ site with only very minor or negligible contribution from other sites. Thus, we find that the $\mathrm{Na} 2 b$ sites inside tri-vacancy clusters sandwiched between $\mathrm{CoO}_{2}$ layers are rattler sites, and the mode at $E \sim 13 \mathrm{meV}$ is a rattling mode.

The $\mathrm{Na} 2 b$ ions also have large-amplitude vibrations in other modes (Fig. 3a). The influence of the rattling mode on the rest of the dispersion can be investigated theoretically by replacing the $\mathrm{Na} 2 b$ ions of atomic mass 23 with fictitious heavier ions of atomic mass 40 . The calculated density of states for these two cases is shown in Fig. 3b. The largest differences between the two curves are found for energies below $E \sim 40 \mathrm{meV}$ and, therefore, the rattling of the $\mathrm{Na} 2 b$ ions in the tri-vacancy clusters is seen to affect mainly these low-energy modes. The density of states is also shown for $\mathrm{NaCoO}_{2}$, and it is clear that the transfer of density from sharp peaks above $E \sim 20 \mathrm{meV}$ to a broad range at low energy will affect $\kappa$.

The atomic displacement parameters for $\mathrm{Na}$ are larger for $\mathrm{Na}_{0.8} \mathrm{CoO}_{2}$ than for $\mathrm{NaCoO}_{2}$, confirming the larger amplitude of vibration in the superstructure (Supplementary Fig. S1). Frozen phonon calculations were performed for the rattler mode, and the potential energy is plotted as a function of displacement in Fig. 3c. The crystallographic asymmetry of the $\mathrm{Na} 2 b$ site gives rise to a cubic term in energy and consequently a significant degree of anharmonicity. This contrasts with symmetric Ce guest rattler modes in skutterudites where the leading term is quartic and the anharmonicity smaller ${ }^{8}$. The energy of this mode is $E \sim 13 \mathrm{meV}$ and, therefore, anharmonicity is expected to become important above $T \sim 150 \mathrm{~K}$.

Measurements of $\kappa$ using a Quantum Design physical property measurement system on a crystal of the same composition are presented in Fig. 4a. The magnitude of $\kappa$, in the region of a few watts per Kelvin-metre, is similar to the values reported for crystals of comparable composition ${ }^{14,15}$. The temperature dependence indicates a contribution from phonon scattering.

To measure phonon linewidths at higher resolution, we have measured a region of the dispersion dominated by a single optical phonon at $\mathbf{Q} \sim(1.01,1.01,1)$ and $E \sim 8 \mathrm{meV}$ using a triple-axis neutron spectrometer. The energy line shape is resolution-limited below $T \sim 80 \mathrm{~K}$, and above this temperature we fitted the data using a Lorentzian convolved with the instrumental resolution function. Figure $4 \mathrm{~b}$ shows a typical energy scan and the phonon lifetimes, $\tau$, related to the energy linewidths, $\Gamma^{\mathrm{ph}}$, by $\tau \sim h / \Gamma^{\mathrm{ph}}$, as a function of temperature. These data are consistent with our measurements averaged over several modes at $T \sim 200 \mathrm{~K}$ using IXS. They are narrow in comparison with previous Raman ${ }^{21}$ and IXS (ref. 23) measurements, presumably owing to the high crystalline quality of our sample. There is no evidence for the marked energy broadening expected for a phonon glass.

The lattice contribution to $\kappa$ was estimated using the expression

$$
\kappa^{\mathrm{L}}=\sum_{j, Q} v_{j x}^{2}(Q) \tau_{j}(Q, T) c_{j}(Q, T)
$$

where $v_{j x}$ is the group velocity along the thermal gradient, $c_{j}$ is the heat capacity of the $j$ th phonon mode, and $\tau_{j}$ is the phonon lifetime. The values of $v_{j x}$ and $c_{j}$ were determined using the calculated dispersions assuming Bose statistics, and the average value of $\tau$ at each temperature was obtained from Fig. $4 \mathrm{~b}$. The estimated value of $\kappa^{L}$ calculated using our model for the square superstructure agrees with the data within experimental uncertainty. The electronic contribution to $\kappa$ estimated using the Wiedemann-Franz law from the electrical conductivity ${ }^{15}$ is an order of magnitude lower.

For comparison, we have estimated $\kappa^{L}$ at $T \sim 200 \mathrm{~K}$ for the stoichiometric compound, assuming (artificially) that $\tau$ is the same (blue circle in Fig. 4a). The fact that this value is roughly six

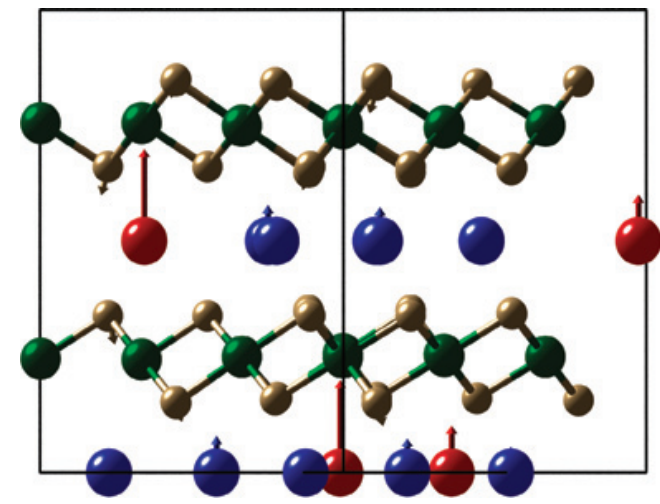

b

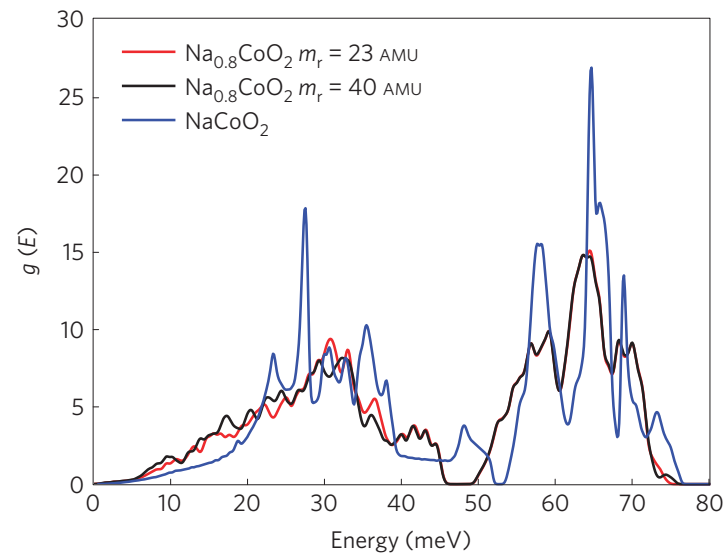

c

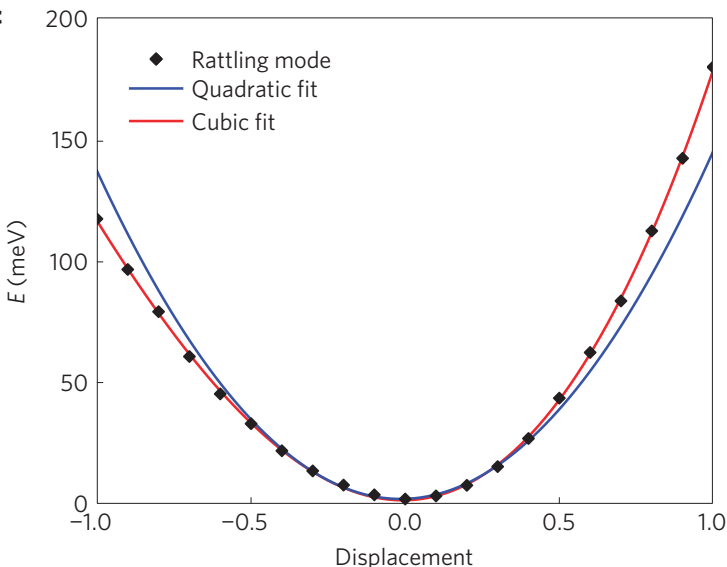

Figure 3 | Influence of rattlers on the rest of the dispersion. a, A typical zone-centre optical phonon, showing how the rattling ions couple strongly to and alter modes. $\mathbf{b}$, The calculated density of states $(g(E))$ for Na rattlers of mass $\left(m_{r}\right) 23$ (red) and for test atoms of mass 40 AMU (black). Significant differences occur below $E \sim 40 \mathrm{meV}$ suggesting that these low-energy modes are strongly affected by the rattling ions. The calculation for $\mathrm{NaCoO}_{2}$ shows that density has been transferred from sharp peaks above $E \sim 20$ meV towards lower-energy modes that contribute strongly to the thermal conductivity. c, Frozen phonon calculation for the rattling mode (black). The displacement is scaled to normal mode amplitude, which corresponds to a maximum in-plane displacement of the $\mathrm{Na} 2 b$ site of $0.3 \AA$. The asymmetry of the potential energy as a function of displacement of the rattling ions clearly demonstrates the anharmonicity of this mode.

times higher clearly indicates that rattling modes are important in lowering $\kappa$, because they contribute significantly to the specific heat but have small propagation velocities. The marked increase in $\kappa$ for the stoichiometric compound is borne out experimentally ${ }^{15}$, where there is an additional factor of two increase over our 
a

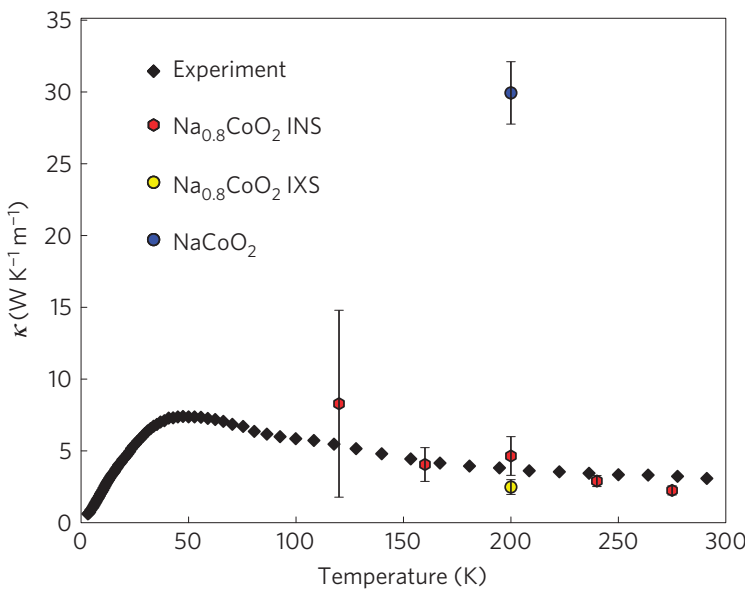

b

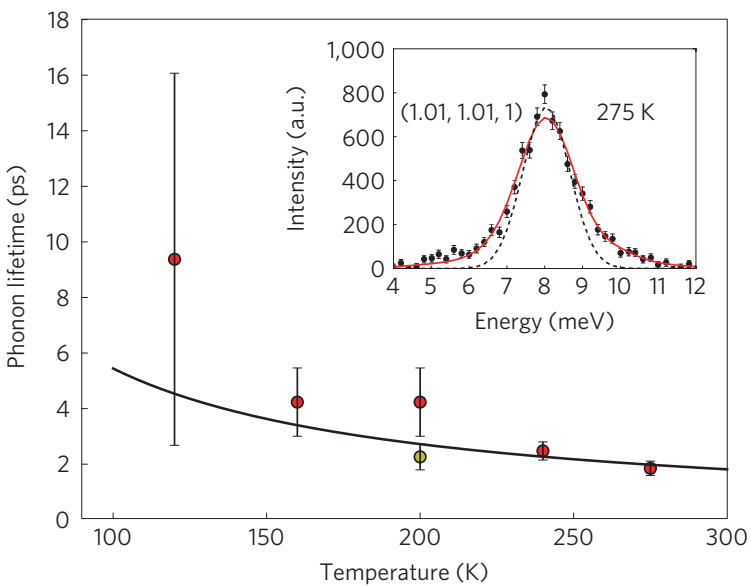

Figure 4 | Thermal properties of sodium cobaltate. a, The measured thermal conductivity of $\mathrm{Na}_{0.8} \mathrm{CoO}_{2}$ (black) is compared with the calculated lattice contribution to the thermal conductivity for the $\mathrm{Na}_{0.8} \mathrm{CoO}_{2}$ superstructure comprising a square array of tri-vacancy clusters using the phonon lifetimes derived using neutrons (red) and X-rays (yellow). The calculated values for $\mathrm{NaCoO}_{2}$ (blue) are a factor of six higher. $\mathbf{b}$, The phonon lifetimes determined from the widths of strong single modes. The lifetimes obtained using INS (red) are consistent with the measurement using IXS (yellow). The solid black line shows the fitted inverse temperature dependence. A typical INS scan at fixed $\mathbf{Q}=(1.01,1.01,1)$ at $T \sim 275 \mathrm{~K}$ is shown in the inset. The red line shows the best fit of a Lorentzian convolved with the resolution Gaussian, and the dashed black line shows the best fit of the resolution Gaussian. Error bars in $\mathbf{a}$ and $\mathbf{b}$ are determined by the fits to phonon lifetimes, and in the inset by Poisson statistics.

calculated value for $\mathrm{NaCoO}_{2}$. This could be attributed to weaker anharmonicity due to the absence of rattling modes, reduced electron-phonon scattering because it is an insulator, or fewer defects in a vacancy-free sample. However, it is clear that the changes to the dispersion from the rattling modes give the dominant contribution to the suppression of $\kappa$.

Multi-vacancy clusters exist over a wide range of concentrations ${ }^{26}$ and, therefore, it is highly likely that they are also responsible for rattling modes throughout the range of compositions relevant to thermoelectric applications ${ }^{15}$. A non-dispersive mode was also observed at a similar energy to our rattling mode in a previous IXS study of $\mathrm{Na}_{0.7} \mathrm{CoO}_{2}$ focused on the softening of Co-related optical branches near the zone boundary and implications for the unconventional superconducting $\operatorname{stat}^{23}$ (Supplementary Fig. S2). The squashing mode for $\mathrm{CoO}_{6}$ octahedra from $E \sim 30-50 \mathrm{meV}$ along $\Gamma \rightarrow \mathrm{M}$ seen in Fig. 2 is ideal for focusing on electronic properties (Supplementary Fig. S3). The low-energy region of the spectrum containing rattling modes is not very sensitive to the hole concentration in the Co layers.

We have performed IXS measurements at $T \sim 320 \mathrm{~K}$ in the disordered stripe phase ${ }^{24}$ and at $T \sim 400 \mathrm{~K}$, where long-range ordering of the superstructure has disappeared. The absence of long-range order prevents $a b$ initio calculations, but we are able to draw some qualitative conclusions. There is renormalization of the phonon dispersion to slightly lower energies and broadening of the energy linewidth by about $50 \%$. It is not clear whether this is due to the increase in disorder, or simply the lattice expansion and additional phonon scattering. The rattling mode persists at high temperature despite the disorder due to the continued presence of multi-vacancy clusters (Supplementary Fig. S4). Thus, the rattling modes are still important at the elevated temperatures relevant to power recovery applications.

Combining experimental studies of phonon dispersions with DFT provides new insights into the factors that control thermal conductivity. In $\mathrm{Na}_{x} \mathrm{CoO}_{2}$ the phonon scattering is mainly intrinsic, and a further challenge would be to calculate the contributions from anharmonicity and electron-phonon scattering to predict phonon lifetimes. These spectroscopic studies directly link first-principles calculations to the design of better thermoelectric materials.

\section{Methods}

Experiment. The IXS crystal of $\mathrm{Na}_{0.8} \mathrm{CoO}_{2}$ was grown using the floating-zone technique at Royal Holloway. A single crystal of size $400 \times 400 \times 20 \mu \mathrm{m}^{3}$ was cleaved from the boule and studied using X-rays at the Mo K-edge at room temperature using an Agilent Xcalibur X-ray diffractometer, and large volumes of reciprocal space were surveyed with a CCD (charge-coupled device) detector. Roughly $80 \%$ of the sample was found to be in the so-called disordered stripe phase ${ }^{22}$. The sample was attached to a glass capillary with silicone grease, and a thin coating of the grease covered the sample to prevent reaction with the atmosphere.

The IXS studies were performed using the ID28 spectrometer at the European Synchrotron Radiation Facility in Grenoble. The sample was placed in a stream of nitrogen gas from a 700 series Oxford Cryostream temperature controller, and at each temperature, the superstructure was determined using a CCD detector. IXS measurements were performed at $T=200,320$ and $400 \mathrm{~K}$, where the sample was found to be predominantly in the square ${ }^{5}$, disordered stripe ${ }^{22}$ and disordered phases, respectively. The sample was aligned accurately using a point detector in two settings with $(h 0 l)$ and $(h h l)$ crystallographic planes horizontal. The lattice parameters were $a=2.844(2)$ and $c=10.803$ (7) for the $P 6_{3} / m m c$ space group at $T=200 \mathrm{~K}$, and the mosaic spread was about $0.5^{\circ}$.

A resolution in energy transfer (full-width at half-maximum) of $3.0 \mathrm{meV}$ was achieved using the $\mathrm{Si}(999)$ reflection for both the monochromator and analyser crystals in backscattering geometry with a Bragg angle of $89.98^{\circ}$. The monochromator beam is focused by a toroidal mirror into a spot size of $250 \times 90 \mu \mathrm{m}^{2}$ (horizontal $\times$ vertical) full-width at half-maximum at the sample position. Energy transfers in the range -20 to $90 \mathrm{meV}$ were measured at fixed $\mathbf{Q}$ by varying the incident energy through the temperature variation of the monochromator crystal, and keeping the final energy fixed. The use of nine spherical analyser crystals allowed simultaneous measurements at multiple values of $\mathbf{Q}$. One of the analysers was chosen to give $\mathbf{Q}$ along high-symmetry directions.

A larger fragment from the same boule of size $7 \times 2 \times 0.5 \mathrm{~mm}^{3}$, with the short direction parallel to the hexagonal $c$-direction, was measured in a Quantum Design physical property measurement system. Gold contacts were bonded to the sample using silver-loaded epoxy adhesive, and the in-plane $\kappa$ was measured over the temperature range $T \sim 2-300 \mathrm{~K}$.

A larger crystal for INS of size $35 \times 10 \times 3 \mathrm{~mm}^{3}$ and of mosaic spread $3^{\circ}$ was screened using the neutron Laue diffractometer SXD at ISIS, and it was found to be a single grain and to adopt the square phase below $T \sim 280 \mathrm{~K}$. It was sealed inside an aluminium can with a helium atmosphere, and mounted in a cryostat with the $(h h l)$ plane horizontal on the triple-axis spectrometer IN8 at the Institut Laue-Langevin. A fixed final energy of $14.7 \mathrm{meV}$ was obtained using a graphite analyser and higher-order contamination was suppressed with a graphite filter after the sample. Energy scans were performed by varying the energy selected by a PG(002) analyser/monochromator, and source-monochromator, monochromator-sample, sample-analyser, analyser-detector collimation 30', 20', 30 ', 30' gave an instrumental energy resolution of full-width at half-maximum $\sim 1.45 \mathrm{meV}$ for the dispersion at $E \sim 8 \mathrm{meV}$ and $\mathbf{Q} \sim(1.01,1.01,1)$.

Theory. The phonon calculations used DFT in the plane-wave pseudopotentia approach as implemented in the CASTEP code ${ }^{25}$ and the spin-polarized Perdew-Burke-Ernzerhof generalized gradient approximation to exchange and correlation $^{27}$. Vanderbilt-type ultrasoft pseudopotentials ${ }^{28}$ were generated within 
CASTEP with small core radii to give very high accuracy and transferability at a plane-wave cutoff of $500 \mathrm{eV}$. Monkhorst-Pack meshes were used to sample the electronic Brillouin zone at a resolution of $0.025 \AA^{-1}$ or finer with a Gaussian smearing of $0.1 \mathrm{eV}$ for the metallic square phase $\mathrm{Na}_{0.8} \mathrm{CoO}_{2}$ calculations. This resulted in a maximum error in forces of $|\delta F|<0.0024 \mathrm{eV}^{-1}$. Lattice dynamics was computed using the $a b$ initio finite displacement/supercell method ${ }^{29}$. A single $10.276 \times 10.276 \times 10.8 \AA^{3}$ cell of the square phase structure containing 114 atoms was sufficient to compute $S(\mathbf{Q}, \omega)$ across the full Brillouin zone of the primitive cell in Fig. 2. Reference calculations excluding the rattler mode were performed in the primitive cell containing 2 formula units of $\mathrm{NaCoO}_{2}$, using a $5 \times 5 \times 2$ supercell for interpolation across the Brillouin zone.

Received 15 November 2012; accepted 24 July 2013; published online 25 August 2013

\section{References}

1. Snyder, G. S. \& Toberer, E. S. Complex thermoelectric materials. Nature Mater. 7, 105-114 (2008).

2. Slack, G. A. in CRC Handbook of Thermoelectrics (ed. Rowe, D. M.) 407-440 (CRC, 1995).

3. Slack, G. A. \& Tsoukala, V. G. Some properties of semiconducting $\operatorname{IrSb}_{3}$. J. Appl. Phys. 76, 1665-1671 (1994).

4. Nolas, G. S. et al. Semiconducting Ge clathrates: Promising candidates for thermoelectric applications. Appl. Phys. Lett. 73, 178-180 (1998).

5. Nolas, G. S. et al. Effect of partial void filling on the lattice thermal conductivity of skutterudites. Phys. Rev. B 58, 164-170 (1998).

6. Toberer, E. S., Baranowski, L. L. \& Dames, C. Advances in thermal conductivity. Annu. Rev. Mater. Res. 42, 179-209 (2012).

7. Roger, M. et al. Patterning of sodium ions and the control of electrons in sodium cobaltate. Nature 445, 631-634 (2007).

8. Feldman, J. L. et al. Lattice dynamics and reduced thermal conductivity of filled skutterudites. Phys. Rev. B 61, R9209-R9212 (2000).

9. Dong, J. et al. Theoretical study of the lattice thermal conductivity in $\mathrm{Ge}$ framework semiconductors. Phys. Rev. Lett. 86, 2361-2364 (2001).

10. Koza, M. et al. Breakdown of phonon glass paradigm in La- and Ce-filled $\mathrm{Fe}_{4} \mathrm{Sb}_{12}$ skutterudites. Nature Mater. 7, 805-810 (2008).

11. Christensen, M. et al. Avoided crossing of rattler modes in thermoelectric materials. Nature Mater. 7, 811-815 (2008)

12. Terasaki, I., Sasago, Y. \& Uchnikora, K. Large thermoelectric power in $\mathrm{NaCo}_{2} \mathrm{O}_{4}$ single crystals. Phys. Rev. B 56, R12685-R12687 (1997).

13. Wang, Y., Rogado, N. S., Cava, R. J. \& Ong, N. P. Spin entropy as the likely source of enhanced thermopower in $\mathrm{Na}_{x} \mathrm{Co}_{2} \mathrm{O}_{4}$. Nature 423 , 425-428 (2003).

14. Foo, M. L. et al. Charge ordering, commensurability, and metallicity in the phase diagram of the layered $\mathrm{Na}_{x} \mathrm{CoO}_{2}$. Phys. Rev. Lett. 92, 247001 (2004).

15. Lee, M. et al. Large enhancement of the thermopower in $\mathrm{Na}_{x} \mathrm{CoO}_{2}$ at high $\mathrm{Na}$ doping. Nature Mater. 5, 537-540 (2006).

16. Xiang, H. J. \& Singh, D. J. Suppression of thermopower of $\mathrm{Na}_{x} \mathrm{CoO}_{2}$ by an external magnetic field: Boltzmann transport combined with spin-polarized density functional theory. Phys. Rev. B 76, 195111 (2007).

17. Li, Z., Yang, J., Hou, J. G. \& Zhu, Q. First-principles lattice dynamics of $\mathrm{NaCoO}_{2}$. Phys. Rev. B 70, 144518 (2004).
18. Jha, P. K. et al. Phonon properties of the intrinsic insulating phase of the cobalt oxide superconductor $\mathrm{NaCoO}_{2}$. Physica B 366, 153-161 (2005).

19. Iliev, M. N. et al. Raman phonons and ageing-related surface disorder in $\mathrm{Na}_{x} \mathrm{CoO}_{2}$. Physica C 402, 239-242 (2004).

20. Lemmens, P. et al. Effect of $\mathrm{Na}$ content and hydration on the excitation spectrum of the cobaltite $\mathrm{Na}_{x} \mathrm{CoO}_{2} \cdot y \mathrm{H}_{2} \mathrm{O}$. J. Phys. Condens. Matter 16, S857-S865 (2004)

21. Shi, Y. G. et al. Raman spectroscopy study of $\mathrm{Na}_{x} \mathrm{CoO}_{2}$ and superconducting $\mathrm{Na}_{x} \mathrm{CoO}_{2} \cdot y \mathrm{H}_{2}$ O. Phys. Rev. B 70, 052502 (2004).

22. Wang, N. L. et al. Infrared probe of the electronic structure and charge dynamics of $\mathrm{Na}_{0.7} \mathrm{CoO}_{2}$. Phys. Rev. Lett. 93, 237007 (2004).

23. Rueff, J-P. et al. Phonon softening in $\mathrm{Na}_{x} \mathrm{CoO}_{2} \cdot y \mathrm{H}_{2} \mathrm{O}$ : Implications for the Fermi surface topology and the superconducting state. Phys. Rev. B 74, R020504 (2006)

24. Morris, D. J. P. et al. Crystal-to-stripe reordering of sodium ions in $\mathrm{Na}_{x} \mathrm{CoO}_{2}$ $(x \geq 0.75)$. Phys. Rev. B 79, R100103 (2009).

25. Clark, S. J. et al. First principles methods using CASTEP. Z. Kristallogr. 220, 567-570 (2005)

26. Hinuma, Y., Meng, Y. S. \& Ceder, G. Temperature-concentration phase diagram of $P 2-\mathrm{Na}_{x} \mathrm{CoO}_{2}$ from first-principles calculations. Phys. Rev. B 77, 224111 (2008).

27. Perdew, J. P. et al. Generalized gradient approximation made simple. Phys. Rev. Lett. 77, 3865-3868 (1996).

28. Vanderbilt, D. Soft self-consistent pseudopotentials in a generalized eigenvalue formalism. Phys. Rev. B 41, 7892-7895 (1990).

29. Frank, W. et al. Ab initio force-constant method for phonon dispersions in alkali metals. Phys. Rev. Lett. 74, 1791-1794 (1995).

\section{Acknowledgements}

We thank the EPSRC for support through grants EP/J011150/1 and EP/J012912/1, and for use of the UK national supercomputing facility HECToR under grant EP/F036809/1. Other calculations used STFC's e-Science facility. We wish to acknowledge the assistance of R. Hanson in developing the crystal structure and normal mode visualizations, which were created using the Jmol software (http://www.jmol.org/).

\section{Author contributions}

Initial planning was by J.P.G., K.R., M.R., A.T.B., A.B. and M.H. The IXS experiment was performed by D.J.V., J.P.G., E.B., L.G. and M.K., and INS measurements were performed by D.J.V., J.P.G., A.P. and M.E. Single crystals were grown by S.U. The thermal conductivity was measured by D.G.P. and the structural characterization was by D.J.V., D.G.P., E.C. and M.J.G. Theoretical modelling was by K.R. and D.J.V. The manuscript was drafted by J.P.G, D.J.V. and K.R. and all authors participated in the writing and review of the final draft.

\section{Additional information}

Supplementary information is available in the online version of the paper. Reprints and permissions information is available online at www.nature.com/reprints. Correspondence and requests for materials should be addressed to J.P.G.

\section{Competing financial interests}

The authors declare no competing financial interests. 